\title{
Multidimensional integral transform involving I-function of several complex variables as kernel
}

\author{
Prathima $\mathbf{J}^{1}$, vasudevan Nambisan T.M ${ }^{2}$ \\ ${ }^{I}$ (Research scholar, SCSVMV, Kanchipuram \& Department of Mathematics, Manipal Institute of Technology/ \\ Manipal University, India) \\ ${ }^{2}$ (Mathematics, SCSVMV University, India)
}

\begin{abstract}
The theory of integral transforms is very useful in solving various types of boundary value problems. By giving various values to the kernel $k(x ; s)$ and considering the interval $(0,1)$ generally, a number of integral transforms have been introduced and studied by several authors from time to time. In the present paper we have introduced multidimensional I-function transform involving I-function of $r$ variables as kernel. In our earlier paper we have established the multiple Mellin transform of product of two I-functions. With the help of that result, in the present paper we have discussed some theorems on multidimensional I-function transform. Special cases include the results involving $\mathrm{H}$-function of several complex variables, I-function of two variables and $\mathrm{H}$ function of two variables. The special case also includes the results given by V.C.Nair.
\end{abstract}

Keywords: Mellin-Barnes integral, H-function, H-function transform, I-function, Multidimensional I-function transform

\section{Introduction}

In 1997, Rathie introduced the generalization of the well-known Fox's H-function [1] which has very recently found interesting applications in wireless communication ([2],[3],[4]). Motivated by the I-function, Shantha Kumari, Nambisan and Rathie introduced I-function of two variables [5] which is a natural generalization of the H-function of two variables introduced earlier by Mittal and Gupta [6] and discussed some of its important properties. Very recently we have introduced and studied an extension of the I-function of several complex variables. This function is defined and represented in the following manner [7].

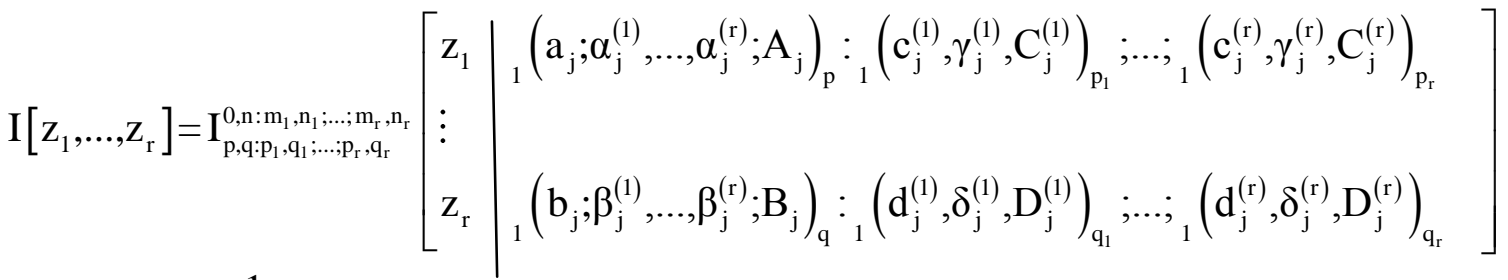

$$
\begin{aligned}
& =\frac{1}{(2 \pi \mathrm{i})^{\mathrm{r}}} \int_{\mathrm{L}_{1}} \ldots \int_{\mathrm{L}_{\mathrm{r}}} \varphi\left(\mathrm{s}_{1}, \ldots, \mathrm{s}_{\mathrm{r}}\right) \theta_{1}\left(\mathrm{~s}_{1}\right) \ldots \theta_{\mathrm{r}}\left(\mathrm{s}_{\mathrm{r}}\right) \mathrm{z}_{1}^{\mathrm{s}_{1}} \ldots \mathrm{z}_{\mathrm{r}}^{\mathrm{s}_{\mathrm{r}}} \mathrm{ds}_{1} \ldots \mathrm{ds_{ \textrm {r } }}
\end{aligned}
$$

where $\varphi\left(s_{1}, \ldots, s_{r}\right)$ and $\theta\left(s_{i}\right), i=1, \ldots, r$ are given by

$$
\varphi\left(s_{1}, \ldots, s_{r}\right)=\frac{\prod_{j=1}^{n} \Gamma^{A_{j}}\left(1-a_{j}+\sum_{i=1}^{r} \alpha_{j}^{(i)} s_{i}\right)}{\prod_{j=n+1}^{p} \Gamma^{A_{j}}\left(a_{j}-\sum_{i=1}^{r} \alpha_{j}^{(i)} s_{i}\right) \prod_{j=1}^{q} \Gamma^{B_{j}}\left(1-b_{j}+\sum_{i=1}^{r} \beta_{j}^{(i)} s_{i}\right)}
$$

$\theta_{i}\left(s_{i}\right)=\frac{\prod_{j=1}^{m_{i}} \Gamma^{D_{j}^{(i)}}\left(d_{j}^{(i)}-\sum_{i=1}^{r} \delta_{j}^{(i)} s_{i}\right) \prod_{j=1}^{n_{i}} \Gamma^{C_{j}^{(i)}}\left(1-c_{j}^{(i)}+\sum_{i=1}^{r} \gamma_{j}^{(i)} s_{i}\right)}{\prod_{j=n_{i}+1}^{p_{i}} \Gamma^{c_{j}^{(i)}}\left(c_{j}^{(i)}-\sum_{i=1}^{r} \gamma_{j}^{(i)} s_{i}\right) \prod_{j=m_{i}+1}^{q_{i}} \Gamma^{D_{j}^{(i)}}\left(1-d_{j}^{(i)}+\sum_{i=1}^{r} \delta_{j}^{(i)} s_{i}\right)}$

Also

$$
z_{i} \neq 0 \text { for } i=1, \ldots, r
$$


- $\quad$ an empty product is interpreted as unity.

- the parameters $m_{j}, n_{j}, p_{j}, q_{j}(j=1, \ldots, r), n, p, q$ are nonnegative integers such that $0 \leq \mathrm{n} \leq \mathrm{p}, \mathrm{q} \geq 0,0 \leq \mathrm{n}_{\mathrm{j}} \leq \mathrm{p}_{\mathrm{j}}, 0 \leq \mathrm{m}_{\mathrm{j}} \leq \mathrm{q}_{\mathrm{j}}(\mathrm{j}=1, \ldots, \mathrm{r})$ ( not all zero simultaneously).

- $\quad \alpha_{j}^{(i)}(j=1, \ldots, p ; i=1, \ldots, r), \beta_{j}^{(i)}(j=1, \ldots, q ; i=1, \ldots, r), \gamma_{j}^{(i)}\left(j=1, \ldots, p_{i} ; i=1, \ldots, r\right), \delta_{j}^{(i)}$ $\left(\mathrm{j}=1, \ldots, \mathrm{q}_{\mathrm{i}} ; \mathrm{i}=1, \ldots, \mathrm{r}\right)$ are assumed to be positive quantities for standardization purpose.

- $\quad a_{\mathrm{j}}(\mathrm{j}=1, \ldots, \mathrm{p}), b_{\mathrm{j}}(\mathrm{j}=1, \ldots, \mathrm{q}), \mathrm{c}_{\mathrm{j}}^{(\mathrm{i})}\left(\mathrm{j}=1, \ldots, \mathrm{p}_{\mathrm{i}} ; \mathrm{i}=1, \ldots, \mathrm{r}\right), \mathrm{d}_{\mathrm{j}}^{(\mathrm{i})}\left(\mathrm{j}=1, \ldots, \mathrm{q}_{\mathrm{i}} ; \mathrm{i}=1, \ldots, \mathrm{r}\right)$ are complex numbers.

- $\quad$ The exponents $A_{j}(j=1, \ldots, p), B_{j}(j=1, \ldots, q), C_{j}^{(i)}\left(j=1, \ldots, p_{i} ; i=1, \ldots, r\right), D_{j}^{(i)}\left(j=1, \ldots, q_{i} ; i=1, \ldots, r\right)$ of various gamma functions involved in (2) and (3) may take non-integer values.

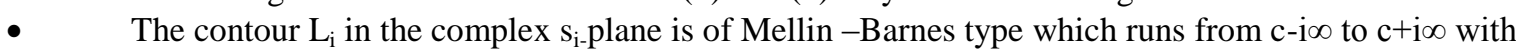
indentations, if necessary, in such a manner that all singularities of $\Gamma^{\mathrm{D}_{\mathrm{j}}^{(\mathrm{i})}}\left(\mathrm{d}_{\mathrm{j}}^{(\mathrm{i})}-\delta_{\mathrm{j}}^{(\mathrm{i})}\right), \mathrm{j}=1, \ldots \mathrm{m}_{\mathrm{i}}$ are to the right and $\Gamma^{c_{j}^{(i)}}\left(1-c_{j}^{(i)}+\gamma_{j}^{(i)}\right), j=1, \ldots, n_{i}$ are to the left of $L_{i}, i=1, \ldots, r$.

Following the results of Braaksma[8] the I-function of ' $r$ ' variables is analytic if

$$
\mu_{i}=\sum_{j=1}^{p} A_{j} \alpha_{j}^{(i)}-\sum_{j=1}^{q} B_{j} \beta_{j}^{(i)}+\sum_{j=1}^{p_{i}} C_{j}^{(i)} \gamma_{j}^{(i)}-\sum_{j=1}^{q_{i}} D_{j}^{(i)} \delta_{j}^{(i)} \leq 0, \quad i=1, \ldots, r
$$

\subsection{Convergence Conditions}

The integral (1) converges absolutely if

$$
\left|\arg \left(\mathrm{z}_{\mathrm{k}}\right)\right|<\frac{1}{2} \Delta_{\mathrm{k}} \pi, \mathrm{k}=1, \ldots, \mathrm{r}
$$

where $\Delta_{\mathrm{k}}=\left[-\sum_{\mathrm{j}=1}^{\mathrm{p}} \mathrm{A}_{\mathrm{j}} \alpha_{\mathrm{j}}^{(\mathrm{k})}-\sum_{\mathrm{j}=1}^{\mathrm{q}} \mathrm{B}_{\mathrm{j}} \beta_{\mathrm{j}}^{(\mathrm{k})}+\sum_{\mathrm{j}=1}^{\mathrm{m}_{\mathrm{k}}} \mathrm{D}_{\mathrm{j}}^{(\mathrm{k})} \delta_{\mathrm{j}}^{(\mathrm{k})}-\sum_{\mathrm{j}=\mathrm{m}_{\mathrm{k}}+1}^{\mathrm{q}_{\mathrm{k}}} \mathrm{D}_{\mathrm{j}}^{(\mathrm{k})} \delta_{\mathrm{j}}^{(\mathrm{k})}+\sum_{\mathrm{j}=1}^{\mathrm{n}_{\mathrm{k}}} \mathrm{C}_{\mathrm{j}}^{(\mathrm{k})} \gamma_{\mathrm{j}}(\mathrm{k})-\sum_{\mathrm{j}=\mathrm{n}_{\mathrm{k}}+1}^{\mathrm{p}_{\mathrm{k}}} \mathrm{C}_{\mathrm{j}}^{(\mathrm{k})} \gamma_{\mathrm{j}}^{(\mathrm{k})}\right]>0$

And if $\left|\arg \left(z_{k}\right)\right|<\frac{1}{2} \Delta_{k} \pi$, and $\Delta_{k}>0, k=1, \ldots, r$ then integral (1) converges absolutely under the following conditions.

(i) $\mu_{k}=0, \Omega_{k}<-1$ where $\mu_{k}$ is given by (4) and

$$
\Omega_{k}=\sum_{j=1}^{p}\left[\frac{1}{2}-R\left(a_{j}\right)\right] A_{j}-\sum_{j=1}^{q}\left[\frac{1}{2}-R\left(b_{j}\right)\right] B_{j}+\sum_{j=1}^{p_{k}}\left[\frac{1}{2}-R\left(c_{j}^{(k)}\right)\right] C_{j}^{(k)}-\sum_{j=1}^{q_{k}}\left[\frac{1}{2}-R\left(d_{j}^{(k)}\right)\right] D_{j}^{(k)}, k=1, \ldots, r
$$

(ii) $\quad \mu_{k} \neq 0$ with $s_{k}=\sigma_{k}+i t_{k}\left(\sigma_{k}\right.$ and $t_{k}$ are real, $\left.k=1, \ldots, r\right), \sigma_{k}$ are so chosen that for $\left|t_{k}\right| \rightarrow \infty$ we have $\Omega_{k}+\sigma_{k} \mu_{k}<-1$

We have discussed the proof of convergent conditions in our earlier paper [7].

\section{Remark 1}

If $D_{j}^{(i)}=1\left(j=1, \ldots, m_{i}, i=1, \ldots, r\right)$ in (1), then the function will be denoted by 


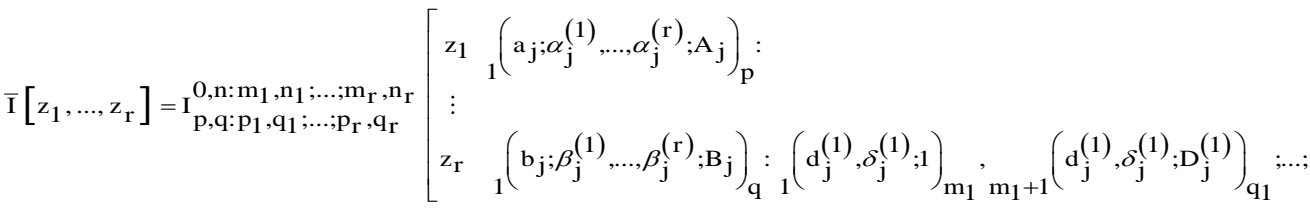

$$
\begin{aligned}
& { }_{1}\left(\mathrm{c}_{\mathbf{j}}^{(1)}, \gamma_{j}^{(1)} ; \mathrm{C}_{\mathrm{j}}^{(1)}\right)_{\mathrm{p}_{1}} ; \ldots ;{ }_{1}\left(\mathrm{c}_{\mathbf{j}}^{(\mathrm{r})}, \gamma_{\mathrm{j}}^{(\mathrm{r})} ; \mathrm{C}_{\mathrm{j}}^{(\mathrm{r})}\right)_{\mathrm{p}_{\mathrm{r}}}
\end{aligned}
$$

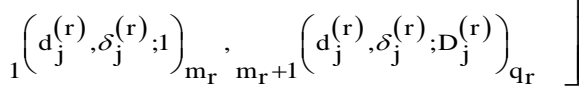

$$
\begin{aligned}
& =\frac{1}{(2 \pi \mathrm{i})^{\mathrm{r}}} \int_{\mathrm{L}_{1}} \ldots \mathrm{L}_{\mathrm{r}} \bar{\theta}_{1}\left(\mathrm{~s}_{1}\right) \ldots \bar{\theta}_{\mathrm{r}}\left(\mathrm{s}_{\mathrm{r}}\right) \varphi\left(\mathrm{s}_{1}, \ldots, \mathrm{s}_{\mathrm{r}}\right) z_{1} \ldots \mathrm{z}_{\mathrm{r}}^{\mathrm{s}_{1}} \mathrm{ds}_{1} \ldots \mathrm{ds} \mathrm{s}_{\mathrm{r}}
\end{aligned}
$$

where

$$
\bar{\theta}_{i}\left(s_{i}\right)=\frac{\prod_{j=1}^{m_{i}} \Gamma\left(d_{j}^{(i)}-\delta_{j}^{(i)} s_{i}\right) \prod_{j=1}^{n_{i}} \Gamma C_{j}^{(i)}\left(1-c_{j}^{(i)}+\gamma_{j}^{(i)} s_{i}\right)}{\prod_{j=m_{i}+1}^{q_{i}} D_{j}^{(i)}\left(1-d_{j}^{(i)}+\delta_{j}^{(i)} s_{i}\right) \prod_{j=n_{i}+1}^{p_{i}} \Gamma_{j}^{(i)}\left(c_{j}^{(i)}-\gamma_{j}^{(i)} s_{i}\right)}, i=1, \ldots, r
$$

\section{Remark 2}

If $C_{j}^{(i)}=1\left(j=1, \ldots, n_{i}, i=1, \ldots, r\right), D_{j}^{(i)}=1\left(j=1, \ldots, m_{i}, i=1, \ldots, r\right)$ and $\mathrm{n}=0$ in (1), then the function will be denoted by

$$
\begin{aligned}
& \bar{I}_{1}\left[z_{1}, \ldots, z_{r}\right]
\end{aligned}
$$

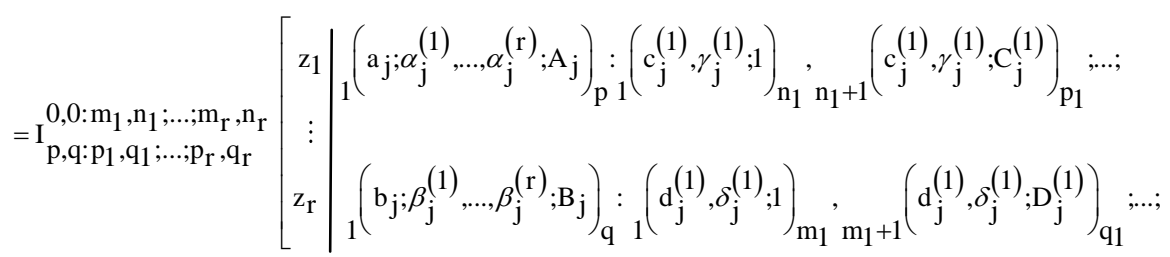

$$
\begin{aligned}
& { }_{1}\left(\mathrm{c}_{\mathrm{j}}^{(\mathrm{r})}, \gamma_{\mathrm{j}}^{(\mathrm{r})} ; 1\right)_{\mathrm{n}_{\mathrm{r}}}, \mathrm{n}_{\mathrm{r}+1}\left(\mathrm{c}_{\mathrm{j}}^{(\mathrm{r})}, \gamma_{\mathrm{j}}^{(\mathrm{r})} ; \mathrm{C}_{\mathrm{j}}^{(\mathrm{r})}\right)_{\mathrm{p}_{\mathrm{r}}} \\
& \left.\left(\mathrm{d}_{\mathrm{j}}^{(\mathrm{r})}, \delta_{\mathrm{j}}^{(\mathrm{r})} ; 1\right)_{\mathrm{m}_{\mathrm{r}}}, \mathrm{m}_{\mathrm{r}+1}\left(\mathrm{~d}_{\mathrm{j}}^{(\mathrm{r})}, \delta_{\mathrm{j}}^{(\mathrm{r})} ; \mathrm{D}_{\mathrm{j}}^{(\mathrm{r})}\right)_{\mathrm{q}_{\mathrm{r}}}\right]
\end{aligned}
$$

where

$$
\begin{gathered}
\varphi_{1}\left(s_{1}, \ldots, s_{r}\right)=\frac{1}{\prod_{j=1}^{q} B_{j}\left(1-b_{j}+\sum_{i=1}^{r} \beta_{j}^{(i)} s_{i}\right) \prod_{j=n+1}^{p} \Gamma A_{j}\left(a_{j}-\sum_{i=1}^{r} \alpha_{j}^{(i)} s_{i}\right)} \\
\bar{\theta}_{i}\left(s_{i}\right)=\frac{\prod_{j=1}^{m_{i}}\left(d_{j}^{(i)}-\delta_{j}^{(i)} s_{i}\right) \prod_{j=1}^{n_{i}} \Gamma\left(1-c_{j}^{(i)}+\gamma_{j}^{(i)} s_{i}\right)}{\prod_{j=m_{i}+1}^{q_{i}} \Gamma_{j}^{(i)}\left(1-d_{j}^{(i)}+\delta_{j}^{(i)} s_{i}\right) \prod_{j=n_{i}+1}^{p_{i}} \Gamma_{j}^{(i)}\left(c_{j}^{(i)}-\gamma_{j}^{(i)} s_{i}\right)} \\
i=1,2, \ldots, r
\end{gathered}
$$

\section{Definition}

Mellin transform of a function $\mathrm{f}(\mathrm{x})$ is defined as

$$
M[f(x) ; s]=\int_{0}^{\infty} x^{s-1} f(x) d x
$$




\section{Result Used}

The following important theorem which is an analogue of the well-known Parseval-Goldstein theorem will be required in the sequel [9]:

Theorem

$$
\text { If } \mathrm{T}\left\{\mathrm{f}_{1}(\mathrm{x}) ; \mathrm{s}\right\}=\varphi_{1}(\mathrm{~s}) \text { and } \mathrm{T}\left\{\mathrm{f}_{2}(\mathrm{x}) ; \mathrm{s}\right\}=\varphi_{2}(\mathrm{~s})
$$

then $\int_{0}^{\infty} f_{1}(x) \varphi_{2}(x) d x=\int_{0}^{\infty} f_{2}(x) \varphi_{1}(x) d x$

(14) provided

that the various integrals involved converge absolutely.

Gupta and Mittal[10] have proved the following theorem.

Uniqueness theorem

If $\mathrm{f}_{1}(\mathrm{x})$ and $\mathrm{f}_{2}(\mathrm{x})$ are continuous for $\mathrm{x} \geq 0$ and

$$
\int_{0}^{\infty} H_{p, q}^{m, n}\left[s x \mid \begin{array}{l}
\left(a_{j} ; \alpha_{j}\right)_{1, p} \\
\left(b_{j} ; \beta_{j}\right)_{1, q}
\end{array}\right] f_{1}(x) d x=\int_{0}^{\infty} H_{p, q}^{m, n}\left[s x \mid \begin{array}{l}
\left(a_{j} ; \alpha_{j}\right)_{1, p} \\
\left(b_{j} ; \beta_{j}\right)_{1, q}
\end{array}\right] f_{2}(x) d x
$$

both integrals being convergent, then $f_{1}(x) \equiv f_{2}(x)$

Without loss of generality the above theorem can be extended to multiple integrals with appropriate convergent conditions.

We have proved the following result [11].

$$
\begin{aligned}
& \int_{0}^{\infty} \ldots \int_{0}^{\infty} \mathrm{x}_{1}^{\rho_{1}-1} \ldots \mathrm{x}_{\mathrm{r}}^{\rho_{\mathrm{r}}-1} \overline{\mathrm{I}}_{1}\left[\mathrm{~s}_{1} \mathrm{x}_{1}^{\lambda_{1}}, \ldots, \mathrm{s}_{\mathrm{r}} \mathrm{x}_{\mathrm{r}}^{\lambda_{\mathrm{r}}}\right] \times \overline{\mathrm{I}}_{1}^{\prime}\left[\mathrm{t}_{1} \mathrm{x}_{1}^{\mu_{1}}, \ldots, \mathrm{t}_{\mathrm{r}} \mathrm{x}_{\mathrm{r}}^{\mu_{\mathrm{r}}}\right] \mathrm{dx} \mathrm{x}_{1} \ldots \mathrm{dx} \mathrm{x}_{\mathrm{r}} \\
& =\frac{1}{\mu_{1} \ldots \mu_{\mathrm{r}}} \mathrm{t}_{1}^{-\frac{\rho_{1}}{\mu_{1}} \ldots}{ }^{-. . \mathrm{t}_{\mathrm{r}}} \frac{\rho_{\mathrm{r}}}{\mu_{\mathrm{r}}} \\
& \mathrm{I}_{\mathrm{p}+\mathrm{q}^{\prime}, \mathrm{p}^{\prime}+\mathrm{q}: \mathrm{p}_{1}+\mathrm{q}_{1}^{\prime}, \mathrm{p}_{1}^{\prime}+\mathrm{q}_{1} ; \ldots ; \mathrm{p}_{\mathrm{r}}+\mathrm{q}_{\mathrm{r}}^{\prime}, \mathrm{p}_{\mathrm{r}}^{\prime}+\mathrm{q}_{\mathrm{r}}}\left[\begin{array}{c|c|c}
\mathrm{s}_{1} \mathrm{t}_{1}{ }^{-\frac{\lambda_{1}}{\mu_{1}}}, \ldots, \mathrm{s}_{\mathrm{r}} \mathrm{t}_{\mathrm{r}} & -\frac{\lambda_{\mathrm{r}}}{\mu_{\mathrm{r}}} & \mathrm{C}: \mathrm{C}_{1} ; \ldots ; \mathrm{C}_{\mathrm{r}} \\
& \mathrm{D}: \mathrm{D}_{1} ; \ldots ; \mathrm{D}_{\mathrm{r}}
\end{array}\right]
\end{aligned}
$$

where

$$
\begin{aligned}
& \mathrm{C}={ }_{1}\left(\mathrm{a}_{\mathrm{j}} ; \alpha_{\mathrm{j}}^{(1)}, \ldots, \alpha_{\mathrm{j}}^{(\mathrm{r})} ; \mathrm{A}_{\mathrm{j}}\right)_{\mathrm{p}},{ }_{1}\left(1-\mathrm{b}_{\mathrm{j}}^{\prime}-\sum_{\mathrm{i}=1}^{\mathrm{r}} \frac{\rho_{\mathrm{i}}}{\mu_{\mathrm{i}}} \beta_{\mathrm{j}}^{(\mathrm{i})}, \frac{\lambda_{1}}{\mu_{1}} \beta_{\mathrm{j}}^{(1)}, \ldots, \frac{\lambda_{\mathrm{r}}}{\mu_{\mathrm{r}}} \beta_{\mathrm{j}}^{(\mathrm{r})} ; \mathrm{B}_{\mathrm{j}}\right)_{\mathrm{q}^{\prime}} \\
& \mathrm{C}_{\mathrm{i}}={ }_{1}\left(\mathrm{c}_{\mathrm{j}}^{(\mathrm{i})}, \gamma_{\mathrm{j}}^{(\mathrm{i})} ; 1\right)_{\mathrm{n}_{\mathrm{i}}},\left(1-\mathrm{d}_{\mathrm{j}}^{\prime(\mathrm{i})}-\delta_{\mathrm{j}}^{(\mathrm{i})} \frac{\rho_{\mathrm{i}}}{\mu_{\mathrm{i}}}, \delta_{\mathrm{j}}^{(\mathrm{i})} \frac{\lambda_{\mathrm{i}}}{\mu_{\mathrm{i}}} ; 1\right)_{\mathrm{m}_{\mathrm{i}}^{\prime}}, \\
& { }_{m_{i}^{\prime}+1}\left(1-d_{j}^{(\mathrm{i})}-\delta_{j}^{\prime(\mathrm{i})} \frac{\rho_{\mathrm{i}}}{\mu_{\mathrm{i}}}, \delta_{\mathrm{j}}^{(\mathrm{i})} \frac{\lambda_{\mathrm{i}}}{\mu_{\mathrm{i}}} ; \mathrm{D}_{\mathrm{j}}^{(\mathrm{i})}\right)_{\mathrm{q}_{\mathrm{i}}}{ }_{\mathrm{n}_{\mathrm{i}}+1}\left(\mathrm{c}_{\mathrm{j}}^{(\mathrm{i})}, \gamma_{\mathrm{j}}^{(\mathrm{i})} ; \mathrm{C}_{\mathrm{j}}^{(\mathrm{i})}\right)_{\mathrm{p}_{\mathrm{i}}}, \mathrm{i}=1, \ldots, \mathrm{r} \\
& \mathrm{D}={ }_{1}\left(\mathrm{~b}_{\mathrm{j}} ; \beta_{\mathrm{j}}^{(1)}, \ldots, \beta_{\mathrm{j}}^{(\mathrm{r})} ; \mathrm{B}_{\mathrm{j}}\right)_{\mathrm{q}},\left(1-\mathrm{a}_{\mathrm{j}}^{\prime}-\sum_{\mathrm{i}=1}^{\mathrm{r}} \frac{\rho_{\mathrm{i}}}{\mu_{\mathrm{i}}} \alpha_{\mathrm{j}}^{(\mathrm{i})}, \frac{\lambda_{1}}{\mu_{1}} \alpha_{\mathrm{j}}^{\prime(1)}, \ldots, \frac{\lambda_{\mathrm{r}}}{\mu_{\mathrm{r}}} \alpha_{\mathrm{j}}^{(\mathrm{r})} ; \mathrm{A}_{\mathrm{j}}^{\prime}\right)_{\mathrm{p}^{\prime}}
\end{aligned}
$$




$$
\begin{aligned}
\mathrm{D}_{\mathrm{i}}= & { }_{1}\left(\mathrm{~d}_{\mathrm{j}}^{(\mathrm{i})}, \delta_{\mathrm{j}}^{(\mathrm{i})} ; 1\right)_{\mathrm{m}_{\mathrm{i}}},\left(1-\mathrm{c}_{\mathrm{j}}^{\prime(\mathrm{i})}-\gamma_{\mathrm{j}}^{(\mathrm{i})} \frac{\rho_{\mathrm{i}}}{\mu_{\mathrm{i}}}, \gamma_{\mathrm{j}}^{\prime(\mathrm{i})} \frac{\lambda_{\mathrm{i}}}{\rho_{\mathrm{i}}} ; 1\right)_{\mathrm{n}_{\mathrm{i}}^{\prime}}, \\
& \left(1-\mathrm{c}_{\mathrm{j}}^{(\mathrm{i})}-\gamma_{\mathrm{j}}^{\prime(\mathrm{i})} \frac{\rho_{\mathrm{i}}}{\mu_{\mathrm{i}}}, \gamma_{\mathrm{j}}^{\prime(\mathrm{i})} \frac{\lambda_{\mathrm{i}}}{\rho_{\mathrm{i}}} ; \mathrm{C}_{\mathrm{j}}^{\prime(\mathrm{i})}\right)_{\mathrm{p}_{\mathrm{i}}^{\prime}} \mathrm{m}_{\mathrm{i}+1}\left(\mathrm{~d}_{\mathrm{j}}^{(\mathrm{i})}, \delta_{\mathrm{j}}^{(\mathrm{i})} ; \mathrm{D}_{\mathrm{j}}^{(\mathrm{i})}\right)_{\mathrm{q}_{\mathrm{i}}}, \quad \mathrm{i}=1, \ldots, \mathrm{r}
\end{aligned}
$$

provided that

(i) $\lambda_{\mathrm{i}}, \mu_{\mathrm{i}}>0, \mathrm{i}=1,2, \ldots, \mathrm{r}$

(ii) $-\lambda_{\mathrm{i}} \min _{1 \leq \mathrm{j} \leq \mathrm{m}_{\mathrm{i}}} \mathfrak{R}\left(\frac{\mathrm{d}_{\mathrm{j}}^{(\mathrm{i})}}{\delta_{\mathrm{j}}^{(\mathrm{i})}}\right)-\mu_{\mathrm{i}} \min _{1 \leq \mathrm{j} \leq \mathrm{m}_{\mathrm{i}}^{\prime}} \mathfrak{R}\left(\frac{\mathrm{d}_{\mathrm{j}}^{(\mathrm{i})}}{\delta_{\mathrm{j}}^{\prime(\mathrm{i})}}\right)<\operatorname{Re}\left(\rho_{\mathrm{i}}\right)$

$$
<\lambda_{\mathrm{i}} \min _{1 \leq \mathrm{j} \leq \mathrm{n}_{\mathrm{i}}} \mathfrak{R}\left(\frac{1-\mathrm{c}_{\mathrm{j}}^{(\mathrm{i})}}{\gamma_{\mathrm{j}}^{(\mathrm{i})}}\right)+\mu_{\mathrm{i}} \min _{1 \leq \mathrm{j} \leq \mathrm{n}_{\mathrm{i}}^{\prime}} \mathfrak{R}\left(\frac{1-\mathrm{c}_{\mathrm{j}}^{\prime(\mathrm{i})}}{\gamma_{\mathrm{j}}^{\prime(\mathrm{i})}}\right), \quad \mathrm{i}=1, \ldots, \mathrm{r} .
$$

and

(iii) the $\overline{\mathrm{I}}_{1}$ - functions involved in (17) exist.

\section{Definition}

In an attempt to generalize the $\mathrm{H}$-function transform we introduced an integral transform whose kernel is the I-function of several complex variables defined by (1). This integral transform is defined and represented in the following manner:

$$
\varphi\left(\mathrm{p}_{1}, \ldots, \mathrm{p}_{\mathrm{r}}\right)=\int_{0}^{\infty} \ldots \int_{0}^{\infty} \overline{\mathrm{I}}_{1}\left[\mathrm{c}_{1}\left(\mathrm{p}_{1} \mathrm{t}_{1}\right)^{\lambda_{1}}, \ldots, \mathrm{c}_{\mathrm{r}}\left(\mathrm{p}_{\mathrm{r}} \mathrm{t}_{\mathrm{r}}\right)^{\lambda_{\mathrm{r}}}\right] \mathrm{f}\left(\mathrm{t}_{1}, \ldots, \mathrm{t}_{\mathrm{r}}\right) \mathrm{dt}_{1} \ldots \mathrm{dt}_{\mathrm{r}}
$$

provided that the integral on the right hand side of (18) is absolutely convergent.

Theorem 1

\section{Some Theorems On Multidimensional I-Function Transform}

$$
\text { If } \varphi\left(\mathrm{p}_{1}, \ldots, \mathrm{p}_{\mathrm{r}}\right)=\int_{0}^{\infty} \ldots \int_{0}^{\infty} \overline{\mathrm{I}}_{1}\left[\mathrm{c}_{1}\left(\mathrm{p}_{1} \mathrm{t}_{1}\right)^{\lambda_{1}}, \ldots, \mathrm{c}_{\mathrm{r}}\left(\mathrm{p}_{\mathrm{r}} \mathrm{t}_{\mathrm{r}}\right)^{\lambda_{\mathrm{r}}}\right] \mathrm{f}\left(\mathrm{t}_{1}, \ldots, \mathrm{t}_{\mathrm{r}}\right) d \mathrm{t}_{1} \ldots \mathrm{dt}_{\mathrm{r}}
$$

and $\psi\left(\mathrm{p}_{1}, \ldots, \mathrm{p}_{\mathrm{r}}\right)=\int_{0}^{\infty} \ldots \int_{0}^{\infty} \overline{\mathrm{I}}_{1}\left[\mathrm{C}_{1}\left(\mathrm{p}_{1} \mathrm{t}_{1}\right)^{\sigma_{1}}, \ldots, \mathrm{C}_{\mathrm{r}}\left(\mathrm{p}_{\mathrm{r}} \mathrm{t}_{\mathrm{r}}\right)^{\sigma_{\mathrm{r}}}\right] \mathrm{f}\left(\mathrm{t}_{1}^{1 / \mathrm{v}_{1}}, \ldots, \mathrm{t}_{\mathrm{r}}^{1 / \mathrm{v}_{\mathrm{r}}}\right) \mathrm{h}\left(\mathrm{t}_{1}, \ldots, \mathrm{t}_{\mathrm{r}}\right) \mathrm{dt}_{1} \ldots \mathrm{dt}_{\mathrm{r}}$

then

$$
\begin{aligned}
& \psi\left(\mathrm{p}_{1}, \ldots, \mathrm{p}_{\mathrm{r}}\right)=\int_{0}^{\infty} \ldots \int_{0}^{\infty} \mathrm{g}\left(\mathrm{x}_{1}, \ldots, \mathrm{x}_{\mathrm{r}}, \mathrm{p}_{1}, \ldots, \mathrm{p}_{\mathrm{r}}\right) \varphi\left(\mathrm{x}_{1}, \ldots, \mathrm{x}_{\mathrm{r}}\right) \mathrm{dx} \mathrm{x}_{1} \ldots \mathrm{dx_{ \textrm {r } }} \\
& \text { where }\left|v_{1} \ldots v_{r}\right| p_{1}^{v_{1}-1} \ldots p_{r}^{v_{r}-1} h\left(p_{1}^{v_{1}}, \ldots, p_{r}^{v_{r}}\right) \bar{I}_{1}\left[C_{1}\left(p_{1}^{v_{1}} k_{1}\right)^{\sigma_{1}}, \ldots, C_{r}\left(p_{r}^{v_{r}} k_{r}\right)^{\sigma_{r}}\right] \\
& =\int_{0}^{\infty} \ldots \int_{0}^{\infty} \mathrm{I}\left[\mathrm{c}_{1}\left(\mathrm{p}_{1} \mathrm{t}_{1}\right)^{\lambda_{1}}, \ldots, \mathrm{c}_{\mathrm{r}}\left(\mathrm{p}_{\mathrm{r}} \mathrm{t}_{\mathrm{r}}\right)^{\lambda_{\mathrm{r}}}\right] \mathrm{g}\left(\mathrm{t}_{1}, \ldots, \mathrm{t}_{\mathrm{r}}, \mathrm{k}_{1}, \ldots, \mathrm{k}_{\mathrm{r}}\right) \mathrm{dt}_{1} \ldots \mathrm{dt}_{\mathrm{r}}
\end{aligned}
$$

provided the integrals involved are absolutely convergent. 
Proof

Using (14) for the transform pairs (19) and (22),

$$
\begin{aligned}
& \int_{0}^{\infty} \ldots \int_{0}^{\infty} g\left(t_{1}, \ldots t_{r}, k_{1}, \ldots, k_{r}\right) \varphi\left(t_{1}, \ldots t_{r}\right) d t_{1} \ldots d t_{r}=\left|v_{1} \ldots v_{r}\right| \int_{0}^{\infty} \ldots \int_{0}^{\infty} t_{1}^{v_{1}-1} \ldots . t_{r}^{v_{r}-1} h\left(t_{1}^{v_{1}} \ldots . t_{r}^{v_{r}}\right) \\
& \overline{\mathrm{I}}_{1}^{\prime}\left[\mathrm{C}_{1}\left(\mathrm{t}_{1}^{\mathrm{v}_{1}} \mathrm{k}_{1}\right)^{\sigma_{1}}, \ldots, \mathrm{C}_{\mathrm{r}}\left(\mathrm{t}_{\mathrm{r}}^{\mathrm{v}_{\mathrm{r}}} \mathrm{k}_{\mathrm{r}}\right)^{\sigma_{\mathrm{r}}}\right] \mathrm{f}\left(\mathrm{t}_{1}, \ldots, \mathrm{t}_{\mathrm{r}}\right) \mathrm{dt}_{1} \ldots \mathrm{dt}_{\mathrm{r}} \\
& \int_{0}^{\infty} \ldots \int_{0}^{\infty} \mathrm{g}\left(x_{1}, \ldots \mathrm{x}_{\mathrm{r}}, \mathrm{p}_{1}, \ldots, \mathrm{p}_{\mathrm{r}}\right) \varphi\left(\mathrm{x}_{1}, \ldots \mathrm{x}_{\mathrm{r}}\right) \mathrm{d} \mathrm{x}_{1} \ldots \mathrm{dx} \mathrm{x}_{\mathrm{r}}=\left|\mathrm{v}_{1} \ldots \mathrm{v}_{\mathrm{r}}\right| \int_{0}^{\infty} \ldots \int_{0}^{\infty} \mathrm{t}_{1}^{\mathrm{v}_{1}-1} \ldots \mathrm{t}_{\mathrm{r}}^{\mathrm{v}_{\mathrm{r}}-1} \mathrm{~h}\left(\mathrm{t}_{1}^{\mathrm{v}_{1}} \ldots \mathrm{t}_{\mathrm{r}}^{\mathrm{v}_{\mathrm{r}}}\right) \\
& \overline{\mathrm{I}}_{1}^{\prime}\left[\mathrm{C}_{1}\left(\mathrm{t}_{1}^{\mathrm{v}_{1}} \mathrm{k}_{1}\right)^{\sigma_{1}}, \ldots, \mathrm{C}_{\mathrm{r}}\left(\mathrm{t}_{\mathrm{r}}^{\mathrm{v}_{\mathrm{r}}} \mathrm{k}_{\mathrm{r}}\right)^{\sigma_{\mathrm{r}}}\right] \mathrm{f}\left(\mathrm{t}_{1}, \ldots, \mathrm{t}_{\mathrm{r}}\right) \mathrm{dt}_{1} \ldots \mathrm{dt}_{\mathrm{r}}
\end{aligned}
$$

Hence,

Putting $t_{i}^{v_{i}}=x_{i}, i=1, \ldots, r$ on the right hand side and using (20) the result follows.

Theorem 2

$$
\begin{aligned}
& \text { If } \varphi\left(\mathrm{p}_{1}^{1 / \mathrm{v}_{1}}, \ldots, \mathrm{p}_{\mathrm{r}}^{1 / \mathrm{v}_{\mathrm{r}}}\right)=\int_{0}^{\infty} \ldots \int_{0}^{\infty} \overline{\mathrm{I}}_{1}\left[\mathrm{c}_{1}\left(\mathrm{p}_{1} \mathrm{t}_{1}\right)^{\lambda_{1}}, \ldots, \mathrm{c}_{\mathrm{r}}\left(\mathrm{p}_{\mathrm{r}} \mathrm{t}_{\mathrm{r}}\right)^{\lambda_{\mathrm{r}}}\right] \mathrm{f}\left(\mathrm{t}_{1}, \ldots, \mathrm{t}_{\mathrm{r}}\right) \mathrm{dt}_{1} \ldots \mathrm{dt}_{\mathrm{r}} \\
& \text { and } \psi\left(\mathrm{p}_{1}, \ldots \mathrm{p}_{\mathrm{r}}\right)=\int_{0}^{\infty} \ldots \int_{0}^{\infty} \mathrm{x}_{1}^{\rho_{1}-1} \ldots \mathrm{x}_{\mathrm{r}}^{\rho_{\mathrm{r}}-1} \overline{\mathrm{I}}_{1}\left(\mathrm{C}_{1}\left(\mathrm{p}_{1} \mathrm{x}_{1}\right)^{\sigma_{1}}, \ldots, \mathrm{C}_{\mathrm{r}}\left(\mathrm{p}_{\mathrm{r}} \mathrm{x}_{\mathrm{r}}\right)^{\sigma_{\mathrm{r}}}\right) \varphi\left(\mathrm{x}_{1}, \ldots, \mathrm{x}_{\mathrm{r}}\right) \mathrm{dx}_{1} \ldots \mathrm{dx_{ \textrm {r } }}
\end{aligned}
$$

then

$$
\begin{aligned}
& \psi\left(p_{1}, \ldots, p_{r}\right)=\frac{1}{\left(v_{1} \ldots v_{r}\right)\left(\lambda_{1} \ldots \lambda_{r}\right)} c_{1}^{-\rho_{1} / v_{1} \lambda_{1}} \ldots c_{r}^{-\rho_{r} / v_{r} \lambda_{r}} \int_{0}^{\infty} \ldots \int_{0}^{\infty} t_{1}^{-\rho_{1} / v_{1}} \ldots t_{r}^{-\rho_{r} / v_{r}}
\end{aligned}
$$

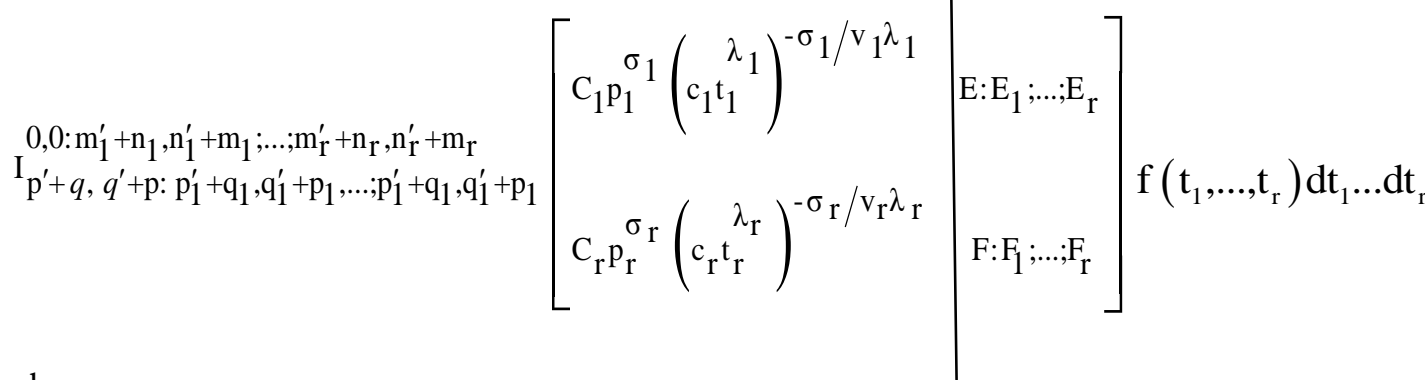

where

$$
\begin{aligned}
& E={ }_{1}\left(\mathrm{a}_{\mathrm{j}}^{\prime} ; \alpha_{\mathrm{j}}^{\prime(1)}, \ldots, \alpha_{\mathrm{j}}^{(\mathrm{r})} ; \mathrm{A}_{\mathrm{j}}^{\prime}\right)_{\mathrm{p}^{\prime}},\left(1-\mathrm{b}_{\mathrm{j}}-\sum_{\mathrm{i}=1}^{\mathrm{r}} \beta_{\mathrm{j}}^{(\mathrm{i})} \frac{\rho_{\mathrm{i}}}{\mathrm{v}_{\mathrm{i}} \lambda_{\mathrm{i}}}, \beta_{\mathrm{j}}^{(1)} \frac{\sigma_{1}}{\mathrm{v}_{1} \lambda_{1}}, \ldots, \beta_{\mathrm{j}}^{(\mathrm{r})} \frac{\sigma_{\mathrm{r}}}{\mathrm{v}_{\mathrm{r}} \lambda_{\mathrm{r}}} ; \mathrm{B}_{\mathrm{j}}\right)_{\mathrm{q}}, \\
& \mathrm{E}_{\mathrm{i}}={ }_{1}\left(\mathrm{c}_{\mathrm{j}}^{(\mathrm{i})}, \gamma_{\mathrm{j}}^{\prime(\mathrm{i})} ; 1\right)_{\mathrm{n}_{\mathrm{i}}^{\prime}},\left(1-\mathrm{d}_{\mathrm{j}}^{(\mathrm{i})}-\delta_{\mathrm{j}}^{(\mathrm{i})} \frac{\rho_{\mathrm{i}}}{\mathrm{v}_{\mathrm{i}} \lambda_{\mathrm{i}}}, \delta_{\mathrm{j}}^{(\mathrm{i})} \frac{\sigma_{\mathrm{i}}}{\mathrm{v}_{\mathrm{i}} \lambda_{\mathrm{i}}} ; 1\right)_{\mathrm{m}_{\mathrm{i}}}, \\
& \left.\left(1-\mathrm{d}_{\mathrm{j}}^{(\mathrm{i})}-\delta_{\mathrm{j}}^{(\mathrm{i})} \frac{\rho_{\mathrm{i}}}{\mathrm{v}_{\mathrm{i}} \lambda_{\mathrm{i}}}, \delta_{\mathrm{j}}^{(\mathrm{i})} \frac{\sigma_{\mathrm{i}}}{\mathrm{v}_{\mathrm{i}} \lambda_{\mathrm{i}}} ; \mathrm{D}_{\mathrm{j}}^{(\mathrm{i})}\right)_{\mathrm{q}_{\mathrm{i}}}\right)_{\mathrm{n}_{\mathrm{i}}^{\prime}+1}\left(\mathrm{c}_{\mathrm{j}}^{\prime(\mathrm{i})}, \gamma_{\mathrm{j}}^{\prime(\mathrm{i})} ; \mathrm{C}_{\mathrm{j}}^{\prime(\mathrm{i})}\right)_{\mathrm{p}_{\mathrm{i}}^{\prime}} \mathrm{i}=1, \ldots, \mathrm{r} \\
& \mathrm{F}={ }_{1}\left(\mathrm{~b}_{\mathrm{j}}^{\prime} ; \beta_{\mathrm{j}}^{\prime(1)}, \ldots, \beta_{\mathrm{j}}^{\prime(\mathrm{r})} ; \mathrm{B}_{\mathrm{j}}^{\prime}\right)_{\mathrm{q}^{\prime}},{ }_{1}\left(1-\mathrm{a}_{\mathrm{j}}-\sum_{\mathrm{i}=1}^{\mathrm{r}} \alpha_{\mathrm{j}}^{(\mathrm{i})} \frac{\rho_{\mathrm{i}}}{\mathrm{v}_{\mathrm{i}} \lambda_{\mathrm{i}}}, \alpha_{\mathrm{j}}^{(1)} \frac{\sigma_{1}}{\mathrm{v}_{1} \lambda_{1}}, \ldots, \alpha_{\mathrm{j}}^{(\mathrm{r})} \frac{\rho_{\mathrm{r}}}{\mathrm{v}_{\mathrm{r}} \lambda_{\mathrm{r}}} ; \mathrm{A}_{\mathrm{j}}\right)_{\mathrm{p}}
\end{aligned}
$$




$$
\mathrm{F}_{\mathrm{i}}={ }_{1}\left(\mathrm{~d}_{\mathrm{j}}^{(\mathrm{i})}, \delta_{\mathrm{j}}^{(\mathrm{i})} ; 1\right)_{\mathrm{m}_{\mathrm{i}}^{\prime}},\left(1-\mathrm{c}_{\mathrm{j}}^{(\mathrm{i})}-\gamma_{\mathrm{j}}^{(\mathrm{i})} \frac{\rho_{\mathrm{i}}}{\mathrm{v}_{\mathrm{i}} \lambda_{\mathrm{i}}}, \gamma_{\mathrm{j}}^{(\mathrm{i})} \frac{\sigma_{\mathrm{i}}}{\mathrm{v}_{\mathrm{i}} \lambda_{\mathrm{i}}} ; 1\right)_{\mathrm{n}_{\mathrm{i}}},
$$

$$
{ }_{n_{i}+1}\left(1-c_{j}^{(i)}-\gamma_{j}^{(i)} \frac{\rho_{i}}{v_{i} \lambda_{i}}, \gamma_{j}^{(i)} \frac{\sigma_{i}}{v_{i} \lambda_{i}} ; C_{j}^{(i)}\right)_{p_{i}}{ }_{m_{i}^{\prime}+1}\left(d_{j}^{\prime(i)}, \delta_{j}^{\prime(i)} ; D_{j}^{\prime(i)}\right)_{q_{i}} \quad i=1, \ldots, r
$$

provided

$$
\sigma_{\mathrm{i}}>0, \lambda_{\mathrm{i}}>0, \Delta>0, \Delta^{\prime}>0
$$

where $\quad \Delta=\sum_{\mathrm{j}=1}^{\mathrm{q}}\left|\prod_{\mathrm{i}=1}^{\mathrm{r}} \beta_{\mathrm{j}}^{(\mathrm{i})}\right|-\sum_{\mathrm{j}=1}^{\mathrm{p}}\left|\prod_{\mathrm{i}=1}^{\mathrm{r}} \alpha_{\mathrm{j}}^{(\mathrm{i})}\right| ; \Delta^{\prime}=\sum_{\mathrm{j}=1}^{\mathrm{q}^{\prime}}\left|\prod_{\mathrm{i}=1}^{\mathrm{r}} \beta_{\mathrm{j}}^{\prime^{(\mathrm{i})}}\right|-\sum_{\mathrm{j}=1}^{\mathrm{p}^{\prime}}\left|\prod_{\mathrm{i}=1}^{\mathrm{r}} \alpha_{\mathrm{j}}^{\prime^{(\mathrm{i})}}\right|$

Proof

On the R.H.S of (24) use (23) to get

$$
\begin{aligned}
\psi\left(\mathrm{p}_{1}, \ldots, \mathrm{p}_{\mathrm{r}}\right)= & \int_{0}^{\infty} \ldots \int_{0}^{\infty}\left\{\int_{0}^{\infty} \ldots \int_{0}^{\infty} \mathrm{x}_{1}^{\rho_{1}-1} \ldots \mathrm{x}_{\mathrm{r}}^{\rho_{\mathrm{r}}-1} \overline{\mathrm{I}}_{1}\left[\mathrm{C}_{1}\left(\mathrm{p}_{1} \mathrm{x}_{1}\right)^{\sigma_{1}}, \ldots, \mathrm{C}_{\mathrm{r}}\left(\mathrm{p}_{\mathrm{r}} \mathrm{x}_{\mathrm{r}}\right)^{\sigma_{\mathrm{r}}}\right]\right. \\
& \left.\times \overline{\mathrm{I}}_{1}\left[\mathrm{c}_{1}\left(\mathrm{x}_{1}^{\mathrm{v}_{1}} \mathrm{t}_{1}\right)^{\lambda_{1}}, \ldots, \mathrm{c}_{\mathrm{r}}\left(\mathrm{x}_{\mathrm{r}}^{\mathrm{v}_{\mathrm{r}}} \mathrm{t}_{\mathrm{r}}\right)^{\lambda_{\mathrm{r}}}\right] \mathrm{dx} \mathrm{x}_{1} \ldots \mathrm{dx} \mathrm{x}_{\mathrm{r}}\right\} \mathrm{f}\left(\mathrm{t}_{1}, \ldots, \mathrm{t}_{\mathrm{r}}\right) \mathrm{dt}_{1} \ldots \mathrm{dt}_{\mathrm{r}}
\end{aligned}
$$

In (26) use (17) to get the R.H.S of (25).

Theorem 3

If $\varphi\left(\mathrm{p}_{1}, \ldots, \mathrm{p}_{\mathrm{r}}\right)=\int_{0}^{\infty} \ldots \int_{0}^{\infty} \overline{\mathrm{I}}_{1}\left[\mathrm{c}_{1}\left(\mathrm{p}_{1} \mathrm{t}_{1}\right)^{\lambda_{1}}, \ldots, \mathrm{c}_{\mathrm{r}}\left(\mathrm{p}_{\mathrm{r}} \mathrm{t}_{\mathrm{r}}\right)^{\lambda_{\mathrm{r}}}\right] \mathrm{f}\left(\mathrm{t}_{1}, \ldots, \mathrm{t}_{\mathrm{r}}\right) d \mathrm{t}_{1} \ldots \mathrm{dt_{ \textrm {r } }}$

and

$$
\psi\left(\mathrm{p}_{1}, \ldots, \mathrm{p}_{\mathrm{r}}\right) \varphi\left(\mathrm{p}_{1}^{\mathrm{v}_{1}}, \ldots, \mathrm{p}_{\mathrm{r}}^{\mathrm{v}_{\mathrm{r}}}\right)=\int_{0}^{\infty} \ldots \int_{0}^{\infty} \overline{\mathrm{I}}_{1}\left[\mathrm{C}_{1}\left(\mathrm{p}_{1} \mathrm{t}_{1}\right)^{\mu_{1}}, \ldots, \mathrm{C}_{\mathrm{r}}\left(\mathrm{p}_{\mathrm{r}} \mathrm{t}_{\mathrm{r}}\right)^{\mu_{\mathrm{r}}}\right] \mathrm{g}\left(\mathrm{t}_{1}, \ldots, \mathrm{t}_{\mathrm{r}}\right) \mathrm{dt}_{1} \ldots \mathrm{dt}_{\mathrm{r}}
$$

then

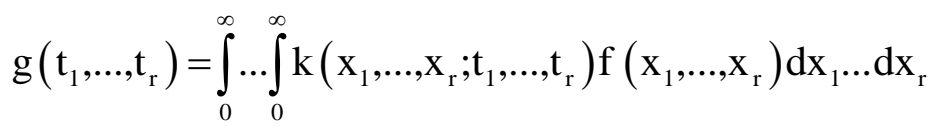

where

$$
\begin{array}{r}
\psi\left(\mathrm{p}_{1}, \ldots, \mathrm{p}_{\mathrm{r}}\right) \overline{\mathrm{I}}_{1}\left[\mathrm{c}_{1}\left(\mathrm{p}_{1}^{\mathrm{v}_{1}} \mathrm{x}_{1}\right)^{\lambda_{1}}, \ldots, \mathrm{c}_{\mathrm{r}}\left(\mathrm{p}_{\mathrm{r}}^{\mathrm{v}_{\mathrm{r}}} \mathrm{x}_{\mathrm{r}}\right)^{\lambda_{\mathrm{r}}}\right]=\int_{0}^{\infty} \ldots \int_{0}^{\infty} \overline{\mathrm{I}}_{1}\left[\mathrm{C}_{1}\left(\mathrm{p}_{1} \mathrm{t}_{1}\right)^{\mu_{1}}, \ldots, \mathrm{C}_{\mathrm{r}}\left(\mathrm{p}_{\mathrm{r}} \mathrm{t}_{\mathrm{r}}\right)^{\mu_{\mathrm{r}}}\right] \\
\mathrm{k}\left(\mathrm{x}_{1}, \ldots, \mathrm{x}_{\mathrm{r}} ; \mathrm{t}_{1}, \ldots, \mathrm{t}_{\mathrm{r}}\right) \mathrm{dt}_{1} \ldots \mathrm{dt}_{\mathrm{r}}
\end{array}
$$

provided the integrals involved are absolutely convergent.

Proof

Multiplying both sides of (30) by $\mathrm{f}\left(\mathrm{x}_{1}, \ldots, \mathrm{x}_{\mathrm{r}}\right)$ and integrating with respect to $\mathrm{x}_{1} \ldots \mathrm{x}_{\mathrm{r}}$ from 0 to $\infty$,

$$
\begin{aligned}
& \psi\left(\mathrm{p}_{1}, \ldots, \mathrm{p}_{\mathrm{r}}\right) \int_{0}^{\infty} \ldots \int_{0}^{\infty} \overline{\mathrm{I}}_{1}\left[\mathrm{c}_{1}\left(\mathrm{p}_{1}^{\mathrm{v}_{1}} \mathrm{x}_{1}\right)^{\lambda_{1}}, \ldots, \mathrm{c}_{\mathrm{r}}\left(\mathrm{p}_{\mathrm{r}}^{\mathrm{v}_{\mathrm{r}}} \mathrm{x}_{\mathrm{r}}\right)^{\lambda_{\mathrm{r}}}\right] \mathrm{f}\left(\mathrm{x}_{1}, \ldots, \mathrm{x}_{\mathrm{r}}\right) \mathrm{dx} \mathrm{x}_{1} \ldots \mathrm{dx} \mathrm{x}_{\mathrm{r}} \\
& =\int_{0}^{\infty} \ldots \int_{0}^{\infty} f\left(x_{1}, \ldots, x_{r}\right)\left\{\int_{0}^{\infty} \ldots \int_{0}^{\infty} \mathrm{k}\left(\mathrm{x}_{1}, \ldots, \mathrm{x}_{\mathrm{r}} ; \mathrm{t}_{1}, \ldots, \mathrm{t}_{\mathrm{r}}\right) \overline{\mathrm{I}}_{1}^{\prime}\left[\mathrm{C}_{1}\left(\mathrm{p}_{1} \mathrm{t}_{1}\right)^{\mu_{1}}, \ldots, \mathrm{C}_{\mathrm{r}}\left(\mathrm{p}_{\mathrm{r}} \mathrm{t}_{\mathrm{r}}\right)^{\mu_{\mathrm{r}}}\right] \mathrm{dt}_{1} \ldots \mathrm{dt}_{\mathrm{r}}\right\} \mathrm{dx}_{1} \ldots \mathrm{dx}_{\mathrm{r}}
\end{aligned}
$$


Using (27), L.H.S of (31) is $\psi\left(\mathrm{p}_{1}, \ldots, \mathrm{p}_{\mathrm{r}}\right) \varphi\left(\mathrm{p}_{1}^{\mathrm{v}_{1}}, \ldots, \mathrm{p}_{\mathrm{r}}^{\mathrm{v}_{\mathrm{r}}}\right)$. Therefore from (17) and (28) we get (29).

The change in the order of integration is valid provided The I- function transform of

$\left|\mathrm{g}\left(\mathrm{t}_{1}, \ldots, \mathrm{t}_{\mathrm{r}}\right)\right|$ and $\mid \mathrm{k}\left(\mathrm{x}_{1}, \ldots, \mathrm{x}_{\mathrm{r}}, \mathrm{t}_{1}, \ldots, \mathrm{t}_{\mathrm{r}} \mid\right.$ exists and the integral (29) is convergent by virtue of De la Vallee Pousein's theorem [12, p-504].

\section{Special Cases}

In above three theorems, if we take $A_{j}(j=1, \ldots, p)=B_{j}(j=1, \ldots, q)=C_{j}^{(i)}\left(j=n_{i}+1, \ldots, p_{i} ; i=1, \ldots, r\right)$

$=D_{j}^{(i)}\left(j=m_{i}+1, \ldots, q_{i} ; i=1, \ldots, r\right)=1$, we get the corresponding results involving $\mathrm{H}$-function of $\mathrm{r}$ variables.

If we put $r=2$ in above three theorems, they reduce to the corresponding results involving I-function of two variables.Further putting all exponents unity we get the results involving $\mathrm{H}$-function of two vcariables.

when $\mathrm{p}=\mathrm{q}=0$ and $\mathrm{r}=1$ in above three theorems we obtain the results given by Nair [13].

\section{References}

Arjun K. Rathie, A new generalization of generalized hypergeometric functions, Le Matematiche, vol. 52, no. 2, 1997, pp. $297-310$.

[1]. I.S.Ansari, F.Yilmaz, M.S.Alouni and O.Kucur, On the sum of gamma random variates with application to the performance of maximal ratio combining over Nakagami-m fading channels,Signal Processing Advances in Wireless Communications (SPAWC), 2012 IEEE 13th International Workshop, 2012, 394398.

[2]. I.S.Ansari,, F.Yilmaz, and M.S.Alouni,On the Sum of Squared eta- Random Variates with Application to the Performance of Wireless Communication Systems, Vehicular Technology Conference (VTC Spring), 2013 IEEE 77th, 2013, 1-6.

[3]. Xia Minghua, Wu Yik-Chung and Aissa Sonia, Exact Outage Probability of Dual-Hop CSI- Assisted AF Relaying Over Nakagami-m Fading Channels, IEEE Transactions on Signal Processing,60(10), 2012, 5578-5583.

[4]. K.Shanthakumari,T.M.Vasudevan Nambisan,K. Arjun K. Rathie, A study of the I-function of two variables,To appear in Le Matematiche. Also see arXiv:1212.6717v1 [math.CV].30Dec2012.

[5]. P.K. Mittal, K.C.Gupta, An integral involving generalized function of two variables, Proc.Indian Acad,Sci.Sect.A32, 1972, $117-$ 123.

[6]. J.Prathima,T.M.Vasudevan Nambisan, K.Shanthakumari, A study of the I-function of several complex variables, International Journal of Engineering Mathematics Volume 2014, Hindawi Publishing Corporation,(2014).

[7]. B.L.J.Braaksma.,Asymptotic expansions and analytic continuations for a class of Barnes- integrals, Compositio Math. 15, 1964, 239-341.

[8]. H.M.Srivastava,K.C.Gupta,S.P.Goyal: The function of one and two variables with applications,(South Asian Publishers, New Delhi, 1982.

[9]. K.C.Gupta, and P.K. Mittal, The H-function transform,IIJ.J.Austral.Math. Univ.Nac.Tucuman Rev.Ser.A-22, 1971, 101-107.

[10]. J.Prathima J, T.M.Vasudevan Nambisan, Multidimensional Mellin transforms involving I-function of several complex variables, International Journal of Mathematics and Statistics Invention,vol.1, 2013, 25-30 .

[11]. T.J.I.A.Bromwich, An introduction to the theory of infinite series (Macmillan and co, London, 1931).

[12]. V.C.Nair, Investigations in transform calculus.,Ph.D thesis,University of Rajasthan, 1968. 